Identity Conditions, Idealisations and Isomorphisms: A Defence of the Semantic Approach

\title{
Steven French
}

School of Philosophy, Religion and History of Science

University of Leeds

\begin{abstract}
In this paper I begin with a recent challenge to the semantic approach and identify an underlying assumption, namely that identity conditions for theories should be provided. I suggest that this demand should be resisted and that the semantic approach should be seen as a philosophical device that we may use to represent certain features of scientific practice. Focussing on the partial structures variant of that approach, I then consider a further challenge that arises from a concern with the role of idealisations in that practice. I argue that the partial structures approach is capable of meeting this challenge and I conclude with some broader observations about the role of such formal accounts within the philosophy of science.
\end{abstract}




\section{Introduction}

Echoing Suppe before him, Halvorson has recently claimed that, '[w]ithin a few short decades, the semantic approach has established itself as the new orthodoxy' (Halvorson, 2012) ${ }^{1}$, before challenging that orthodoxy. In what follows, I shall begin by outlining what I see as the basis for that challenge, which lies in the demand that identity conditions for theories be provided, before responding to it in terms of the partial structures variant of the semantic approach. I will then consider a further challenge to this approach that arises from a concern with idealisations and will indicate how this can also be dealt with. I shall conclude with some broader observations about the role of such formal accounts within the philosophy of science.

\section{Identity Conditions and the Challenge to the Semantic Approach}

In challenging the orthodoxy, Halvorson repeatedly insists that what is at issue here is the identity of theories (indeed, the whole thrust of his paper is encapsulated in section 4, which is entitled 'Identity Crisis for Theories'). Thus he writes, '[a]ccording to the semantic view, a theory is [my emphasis] a class of models' (2012 p. 190; later on he talks of the semantic view 'reducing' theories to sets of models; ibid., p. 192) and his aim is explicit, namely that '... it will become clear that it is impossible to formulate good identity criteria for theories when they are considered as classes of models.' (ibid. p. 190; see also p. 201) ${ }^{2}$ Indeed, the discussion throughout is presented in terms of individuating theories but of course, framing the debate over the viability of the semantic approach in this way leads to the possibility of question begging over what counts as 'the same' theory to begin with. Thus, to demonstrate that the semantic approach identifies theories that should be regarded as distinct, Halvorson's strategy is to syntactically formulate a couple of theories, show that they are inequivalent by the standard criterion of definitional equivalence and then point out that the relevant sets of models are isomorphic and hence the theories must be counted as the same according to the approach and contrary to how they should be understood.

As Glymour has noted, one could respond by insisting that this is question begging in the following sense: the question of what 'is' the theory is precisely what is in dispute, so to maintain that a theory 'is' its syntactic formulation in terms of which it can be shown to be inequivalent to another, which the semantic approach renders as equivalent, is precisely to beg the question against the latter view (Glymour 2013, p. 287). Glymour himself thinks this objection doesn't go through because of the role of language within the semantic approach itself: to present a theory as a class of relational structures is to describe that class in some language (ibid.). But that misses the point. The advocate of the semantic approach could acknowledge the need for some such description but maintain that the role of language is trivial or, at the very least, should be downplayed and that if we are to seek identity criteria for theories it should be in model-theoretic

\footnotetext{
${ }^{1}$ cf. also Frigg (2006, p. 51) and LeBihan (2012) who also refer to the Semantc approach as the orthodox view of theories and models. And here is Suppe from the late 1980s: "The Semantic Conception of Theories today probably is the philosophical analysis of the nature of theories most widely held among philosophers of science" (Suppe, 1989, p. 3).

2 Basically, by demonstrating how certain proposals for defining an isomorphism fail.
} 
terms (cf. van Fraassen 1989, p. 222). On that basis, the demonstration of interdefinability between two syntactically formulated objects would indeed be irrelevant (Glymour op. cit.).

Furthermore, and in similar vein, the examples of 'theories' presented in this exchange between Halvorson and Glymour are either 'toy' logic cases or taken from mathematics where, in both cases, clearly articulated formulations can be given in terms of which equivalence, or not, can be explicitly demonstrated via some standard technical device and then contrasted with the relevant relationship obtained via the relevant such device at the level of classes of relational structures. In such cases, and leaving aside the above issue of question begging, the identity criteria of the theories can be made clear, at one level or another. But this is typically not the case when it comes to examples of scientific theories. Should Newton's theory of mechanics or Maxwell's theory of electrodynamics or Einstein's General Relativity be identified with certain syntactic formulations? To do so would clearly beg the question against the semantic approach and in these cases we don't have the clearly articulated formulations that Halvorson presents. Instead we have ... well, that's a good question actually and one that deserves a more developed answer than I can give here but for now lets say that we have some equations, interpreted of course, written down in various texts, in various languages, sometimes 'expressed' or presented in quite different ways. We could, of course, attempt to construct a syntactic formulation of any or all of these theories, along the lines of the socalled Received View of theories but to again, to insist that that formulation is the theory and that in such terms the semantic approach misidentifies it, is of course, and precisely, to beg the question. (And equally, the proponent of the Received View can say the same if we were to articulate the criteria of theory identification in model-theoretic terms!)

The point is that we don't have the nice clear and clean examples that the above debate focuses on. What we have is something a lot more complex and a lot messier, in the context of which the articulation of criteria of theory identification is a much less straightforward and much more contentious business. As a result, we, as philosophers of science, then have to decide on what basis we are going to select those features of this messy collection of statements and diagrams, supposed axioms that don't look like anything you were taught in logic class, equations and claims, that we then take to be 'the' theory in question. One answer - drawn from the recent developments of the semantic approach-is to focus on the representational role of these scientific models.

Thus, van Fraassen, in also responding to Halvorson and also dragging the debate back into the context in which the semantic approach was originally proposed, namely that of scientific theories, writes that when a scientist presents a theory '... she provides a class of models for the representation of those phenomena' (2014, p. 277). Of course, that immediately raises the further issue of determining the characteristics of representation by which we may pick out scientific representations from the melee that is scientific practice in any field. Again, there is more to say (see French forthcoming) but here van Fraassen draws the time honoured comparison with representation in art: '... we properly 
speak of a model of combustion or of the San Francisco Bay in the way we speak of a painting of fire or of the Giaconda. ' $\left(2014\right.$, p. 277) ${ }^{3}$

Given, then, that scientific models are, primarily, representations, in what sense may they also be mathematical structures in the way that the semantic approach proposes? The answer is straightforward: 'A model is a mathematical structure in the same sense that the Mona Lisa is a painted piece of wood.' (ibid.). In other words, both the representational content of the painting and the actual painted piece of wood are what make the Mona Lisa the artefact that it is, and similarly, there is more to a model, as a scientific artefact, than the relational structure in terms of which we can define embeddability, isomorphism and so on. In particular, if we restrict our considerations to the former, and take a model to be a structure plus an interpretation which maps expressions in some language to elements of that structure, so that sentences may come out true under such an interpretation, we stand to overlook the representational aspect that is so crucial in the scientific context.

Indeed, Thomson-Jones argues that that not only should we keep these two roles - the truth-making and the representational - distinct, we should drop the former from our characterisation of the semantic approach entirely $(2006)^{4}$. His principal motivation for this view is that,

'When it comes to showing the naturalness and plausibility with which theories in the empirical sciences can be viewed as collections of models ... it is quite unclear that the models in question are, as constituents of those theories, functioning as truth-making structures in any substantial way.' (ibid., p. 530).

Even if we eschew the kinds of toy examples that Halvorson favours, and consider, for example, Suppes' presentation of Newtonian mechanics via the appropriate set-theoretical predicate, a model taken from the collection picked out by this predicate is a truth-making structure for the relevant statements only in the 'thin' sense that it provides a domain of discourse for the quantifiers featuring in these sentences. And this is because the latter are not, of course, uninterpreted and requiring interpretation in the way that a Tarski-type model provides an interpretation for some sentence of a first-order language. On the contrary, they are already interpreted sentences of 'mathematical English. So, the model picked out by the set-theoretic predicate is not a 'serious interpreter' of these sentences but only a 'description fitter' (ibid., p. 531).

What the set-theoretic predicate provides us with, then, is ' ... a perfectly good tool for picking out a collection of mathematical models' (ibid., p. 532). And the representational character of the latter is precisely what the advocates of the Semantic Approach need to focus on if they are to maintain the aversion to all the linguistic issues besetting the Received View and stay close to scientific practice (ibid., pp. 533-534). Indeed, Thomson-Jones argues, shifting this focus yields a much more flexible form of Semantic Approach since the many kinds of mathematical structures and concomitant different ways they can serve

\footnotetext{
3 For further on modelling San Francisco bay, see Weisberg 2013.

${ }^{4}$ Again, he frames the debate in terms of identifying scientific theories with objects of a certain sort, namely models and distinguishes two broad versions of the Semantic Approach: the stronger which takes a scientific theory to be a collection of models and a weaker form that takes it as 'best thought of' as such a collection (2006, p. 529).
} 
representational ends, puts a 'rich palette at our disposal' when it comes to understanding scientific practice.(ibid., p. 534).

Likewise, van Fraassen observes that we could, rather perversely perhaps, adopt a kind of Received View stance towards the philosophy of art and rationally reconstruct the Mona Lisa in terms of a mapping from certain natural language expressions to features of the painted piece of wood, such that certain statements made in artbooks, say, come out true under that interpretation, but this is just as un-illuminating when it comes to artistic practice as adopting the above stance towards scientific representations (op. cit., p. 278). In both cases, it is more natural to point to the painting or the scientific representation and say 'that is the Mona Lisa/Newtonian mechanics (respectively)' ${ }^{5}$

The upshot then, is that given that a scientific model is a representation, '...it does not follow that the identity of a theory can be defined in terms of the corresponding set of mathematical structures without reference to their representational function. ' (ibid., p. 278). And if we focus only on such structures while ignoring the representational function then of course we will identify putative theories that are distinct - but we always knew that, as the well-known examples of the equations describing gas diffusion and temperature distributions over time demonstrate (ibid., p. 279). It is only by appreciating their distinct representational functions that we can see that they are not the same, even if the relevant mathematical structures are.

Here we might recall Halvorson's suggestion that, '... the semantic view was not wrong to treat theories as collections of models; rather, it was wrong to treat theories as nothing more than collections of models.' (2012, p. 204). However, the point is that it is Halvorson's conception that is wrong. As French and Saatsi noted some years ago, '[i]t seems to be a popular misconception of the semantic view that it says nothing but the following about theories: theories are (with 'is' of identity) just structures (models).' (2006, p. 552). Dropping that misconception would go a long way towards helping to develop a more nuanced approach to how we, philosophers of science, should represent, for our own purposes, the elements of practice that we are concerned with.

\section{Isomorphisms: Partial and Otherwise}

Such an approach can be articulated within the framework of the partial structures variant of the Semantic Approach, or so it has long been maintained (da Costa and French 2003). The formal details have been given many times elsewhere (ibid.), but in summary are as follows ${ }^{6}$ :

A partial structure is a set-theoretic construct $A=\left\langle D, R_{i}\right\rangle_{i \in l}$, where $D$ is a non-empty set and each $R_{i}$ is a partial relation. A partial relation $R_{i}$ over $D$ is a relation that is not necessarily defined for all $n$-tuples of elements of $D$ (see da Costa and French 1990, p. 255). Each partial relation $R$ can be viewed as an ordered triple $\left\langle R_{1}, R_{2}, R_{3}\right\rangle$, where $R_{1}, R_{2}$, and $R_{3}$ are mutually disjoint sets, with $R_{1}$ $\cup R_{2} \cup R_{3}=D^{n}$, and such that: $R_{1}$ is the set of $n$-tuples that (we take to) belong to

\footnotetext{
${ }^{5}$ Of course, things are not quite that simple; see French forthcoming.

${ }^{6}$ It is assume that we are working in Zermelo-Fraenkel set theory (with the axiom of choice), with its familiar first-order language (see, e.g., Jech 2006).
} 
$R ; R_{2}$ is the set of $n$-tuples that (we take) do not belong to $R$, and $R_{3}$ is the set of $n$ tuples for which it is not defined whether they belong or not to $R .^{7}$

If we have two partial structures, $A=\left\langle D, R_{k}\right\rangle_{k \in K}$ and $A^{\prime}=\left\langle D^{\prime}, R_{k}^{\prime}\right\rangle_{k \in K}$

(where $R_{k}$ and $R_{k}^{\prime}$ are partial relations as above, so that $R_{k}=<R_{k 1}, R_{k 2}, R_{k 3}>$ and $R_{k}^{\prime}$ $=<R_{k 1}^{\prime}, R_{k 2}^{\prime}, R_{k 3}^{\prime}>$ ), then a (partial) function $f$ from $D$ to $D^{\prime}$ is a partial isomorphism between $A$ and $A^{\prime}$ if (a) $f$ is bijective, and (b) for all $x$ and $y$ in $D$, $R_{k 1} x y \leftrightarrow R^{\prime}{ }_{k 1} f(x) f(y)$ and $R_{k 2} x y \leftrightarrow R^{\prime}{ }_{k 2} f(x) f(y)$ (French and Ladyman 1999; Bueno 1997)..$^{8}$ Of course, if $R_{k 3}=R_{k 3}^{\prime}=\varnothing$, so that we no longer have partial structures but 'total' ones, then we recover the standard notion of isomorphism (see Bueno 1997).

Furthermore, we say that a (partial) function $f: D \rightarrow D^{\prime}$ is a partial homomorphism from $A$ to $A^{\prime}$ if for every $x$ and every $y$ in $D, R_{k 1} x y \rightarrow R_{k 1}^{\prime} f(x) f(y)$ and $R_{k 2} x y \rightarrow R_{k 2}^{\prime} f(x) f(y)$ (Bueno, French, and Ladyman 2002). Again, if $R_{k 3}$ and $R_{k}{ }^{\prime} 3$ are empty, we obtain the standard notion of homomorphism as a particular case.

Using this formalism, we can also represent the hierarchy of modelswhat Suppes called models of data, of instrumentation, of experiment (Suppes 1962), as well as various kinds of 'intermediate' models - that take us from the phenomena to the theoretical level (Bueno1997, 600-6021):

Let $S=\left\langle D, R_{i}\right\rangle_{i \in I}$ and $S^{\prime}=\left\langle D^{\prime}, R_{i}^{\prime}\right\rangle_{i \in I}$ be partial structures. So, each $R_{i}$ is of the form $\left\langle R_{1}, R_{2}, R_{3}\right\rangle$, and each $R_{i}^{\prime}$ of the form $\left\langle R_{1}^{\prime}, R_{2}^{\prime}, R_{3}^{\prime}\right\rangle$.

We say that a partial function $f: D \rightarrow D^{\prime}$ is a partial isomorphism between $S$ and $S^{\prime}$ if (i) $f$ is bijective, and (ii) for every $x$ and $y \in D, R_{1} x y \leftrightarrow R^{\prime} f(x) f(y)$ and $R_{2} x y$ $\leftrightarrow R_{2}^{\prime} f(x) f(y)$. So, when $R_{3}$ and $R_{3}^{\prime}$ are empty (that is, when we are considering total structures), we have the standard notion of isomorphism.

In terms of this framework, we can also represent the hierarchy of models (Suppes 1962) - models of data, of instrumentation, of experiment-that take us from the phenomena to the theoretical level (Bueno 1997, 593-607):

$$
\begin{aligned}
& A_{k}=\left\langle D_{k}, R_{k 1}, R_{k 2}, R_{k 3}, \ldots, R_{k n}\right\rangle \\
& A_{k-1}=\left\langle D_{k-1}, R_{(k-1) 1}, R_{(k-1) 2}, R_{(k-1) 3}, \ldots, R_{(k-1) n}\right\rangle \\
& \ldots \\
& A_{3}=\left\langle D_{3}, R_{31}, R_{32}, R_{33}, \ldots, R_{3 n}\right\rangle \\
& A_{2}=\left\langle D_{2}, R_{21}, R_{22}, R_{23}, \ldots, R_{2 n}\right\rangle \\
& A_{1}=\left\langle D_{1}, R_{11}, R_{12}, R_{13}, \ldots, R_{1 n}\right\rangle
\end{aligned}
$$

where each $R_{i j}$ is a partial relation of the form $\left\langle R_{i j}{ }^{1}, R_{i j}{ }^{2}, R_{i j}{ }^{3}\right\rangle-$ with $R_{i j}{ }^{1}$ representing the $n$-tuples that belong to $R_{i j}, R_{i j}{ }^{2}$, the ones that do not belong to $R_{i j}$, and $R_{i j}{ }^{3}$, those for which it is not defined whether they belong or not $\longrightarrow$ such that, for every $i, 1 \leq i \leq k, \operatorname{card}\left(R_{i j}{ }^{3}\right)>\operatorname{card}\left(R_{(i+1) j^{3}}\right)$ (Bueno ibid., 600-603). The partial relations are extended as one goes up the hierarchy, in the sense that at each

\footnotetext{
7 To avoid a possible confusion between $R_{1}, R_{2}$, and $R_{3}$ and particular occurrences of a partial relation $R_{i}$, we will always refer to the former as $R_{1^{-}}, R_{2^{-}}$and $R_{3}$-components of the partial relation $R_{i}$.

${ }^{8}$ For simplicity, we are considering here only two-place relations. But the definition can, of course, be easily extended to $n$-place relations.
} 
level, partial relations which were not defined at a lower level come to be defined, with their elements belonging to either $R_{1}$ or $R_{2} \cdot{ }^{9}$

Using this framework, a notion of partial or quasi-truth (see, e.g., da Costa and French 2003) can be defined as follows: If $B$ is a total structure, whose relations of arity $n$ are defined for all $n$-tuples of elements of its universe, and if $P$ is a set of accepted sentences, ${ }^{10}$ then $B$ is said to be $A$-normal if:

(i) the universe of $B$ is $A$;

(ii) the relations of $B$ extend the corresponding partial relations of $A$;

(iii) if $c$ is an individual constant, then $c$ is interpreted by the same element in both $A$ and $B$;

(iv) if $s \in P$, then $B \mid=s$ (where ' $\mid=$ ' stands for the logical consequence relation in the Tarskian sense).

That is, loosely speaking, a total structure $B$ is called $A$-normal if it has the same similarity type as $A$, its relations extend the corresponding partial relations of $A$, and the sentences of $P$ are true, in the Tarskian sense, in $B$. Then a sentence $s$ is said to be quasi-true in a partial structure $A$, or in the domain $D$ that $A$ partially reflects, if there is an $A$-normal structure $B$ and $s$ is true in the Tarskian sense in $B$. Clearly, $A$ is not conceived of as reflecting the (total) structure of $D$, but as only partially mirroring this domain. Thus the partial structure $A$ has to capture some fundamental aspects of $D$, or some 'elements of truth', although it does not mirror $D$ perfectly. For simplicity, one can say that a sentence $s$ is quasi-true if there is a partial structure $A$ and a corresponding $A$-normal structure $B$ in which $s$ is true (in the Tarskian sense). If $s$ is not quasi-true (in a partial structure $A$ according to an $A$-normal structure $B$ ), then $s$ is said to be quasi-false (in $A$ according to $B$ ).

The claim that has been made is that this formalism offers a useful framework for capturing and thereby representing, at the level of the philosophy of science, scientific practice. Note, however, that I am not claiming that theories are partial structures, or at least, not in any meaningful -that is, ontological sense. If we accept that models and theories are not the kinds of 'things' that have identity conditions then the 'syntactic' approach, the 'semantic' approach and other such approaches can be seen to be meta-level devices that we, as philosophers of science, can deploy in order to represent those aspects of scientific practice that we are interested in (see also French $2014 \mathrm{Ch} .5$ and forthcoming). Of course, some of those aspects or features are referred to as theories and models by scientists themselves but we should be careful not to take that as indicative of some clearly delineated 'thing' whose identity we can definitively pin down. Take Maxwell's electrodynamics for example - as Vickers has persuasively argued, the debate over whether it is formally inconsistent or not turns precisely on the issue of what the theory is taken to be (Vickers 2013). Even classical mechanics in general is problematic, as the debate regarding the priority of Hamiltonian over Lagrangian formulations (or vice versa) reveals (North 2009; Curiel 2014). Indeed, the question 'what is classical physics?',

\footnotetext{
${ }^{9}$ For an additional application of this framework to the idea of partial conceptual spaces, see Bueno 2016.

10 This set of accepted sentences $P$ represents the accepted information about the structure's domain. Depending on the interpretation of science that is adopted, different kinds of sentences are to be introduced in $P$ : realists will typically include laws and theories, whereas empiricists will add mainly certain regularities and observational statements about the domain in question.
} 
forms the jumping off point for a recent historical analysis of the distinction between 'classical' and 'modern' physics (Gooday and Mitchell 2013) that concludes that in fact the distinction emerged over a long period of time, extending into the 1930s, and is dependent on the geographical location considered. And of course, classical continuum mechanics is famously inhomogeneous, presenting a complex patchwork of models, principles, approximations and so forth (Wilson 2014). Things get even worse when we move to the quantum context: which version of quantum field theory should we take as canonical, the rigorously axiomatised one that doesn't apply to any actual system, or the one that does but is likewise a hodge-podge of models and techniques at best, inconsistent at worst (see the debate between Fraser (2011) and Wallace (2011)). There is more to say about this, of course but any insistence that science itself presents clearly identifiable and delineable theories breaks down once close attention is paid to the history and the practice.

My suggestion, then, is that the partial structures approach offers a useful - and I would argue, the best! - (meta-level) representation of the relevant features of those scientific practices. precisely because of the way it can formally accommodate the open ended nature of those features of such practices that we call theories and models. One of the problems with specifying identity conditions is that the relevant features are typically not static - 'theories' not only change and develop over time but also 'draw' on further features from both other such 'theories' or features of other practices, as well as from mathematics of course (da Costa and French 2003; see also Bueno and French forthcoming). ${ }^{11}$ Furthermore, once we, as philosophers of science have determined what we are taking to be a given a theory or set of models (perhaps drawing on the views of the science communities involved), with partial isomorphisms understood as holding both 'horizontally' and 'vertically' between such features as for example, data models and theoretical models, it can also capture the multitude of relationships that constitute scientific progammes in general (Bueno 1997 and 2000; da Costa and French op cit.). Further extended again to include partial homomorphisms, it can also accommodate the relationships between such features and the mathematics in which they are 'framed', thus capturing the apploicability of mathematics to science (Bueno, French and Ladyman 2002; see also Bueno and French 2012); in particular, this approach can capture the 'surplus structure' of mathematics, which of course plays an important heuristic role in scientific developments (Bueno and French forthcoming).

However, it has been argued that the partial structures framework cannot capture a crucial feature of these scientific practices, namely idealisations. The rest of this paper will be taken up with a consideration of and response to this criticism.

\section{Idealisations and Partial Structures}

Here I shall begin by focussing on Weisberg's recent analysis of modelling and simulations in science, not least because he offers a more exact set of criteria, which he feels any account of scientific modelling must meet, than is usually

\footnotetext{
${ }^{11}$ And of course it is precisely such considerations that motivate a shift from theories to 'research programmes'.
} 
found in the literature. In that context he argues that the partial structures approach fails to satisfy these criteria ${ }^{12}$. In particular, he insists, since models typically involve idealizations of various kinds, any account of the model-world relationship should be able to '... distinguish more successful instances of representation from less successful ones.' (Weisberg 2013, p. 136) - this is the 'idealization' criterion. Furthermore, the model-world relationship should be 'scalar', in the sense that the relationship should be regarded as coming in degrees and should be representable on a scale since '... models can tell us a greater or lesser number of true things depending on their degree of idealization ...' (ibid.). It should also allow for 'qualitative' as well as quantitative comparisons and also for the model-world relationship to depend on 'context' and in case where contexts of use differ, for example, it should also accommodate the 'adjudication' of extra-empirical disagreements regarding how good the model is ${ }^{13}$.

Now, although Weisberg acknowledges that the partial structures approach represents a step in the right direction, he insists that it fails to meet these 'idealization', 'scalar', 'qualitativeness' and 'context dependence' criteria. So, let's begin with the first and consider what it would mean for a model, characterised set-theoretically as above, to be idealised or to contain idealisations. Pincock (2005) suggests two conditions that must be met: (i) there is no isomorphism relating it to the situation that it purportedly represents and (ii) the relevant agents are aware of this (ibid., p. 1253). Thus, as Weisberg spells out, isomorphism based accounts straightforwardly fail to meet this desideratum (op. cit. pp. 137-138) because they require all the elements of the model to correspond to elements of the relevant system. But consider the ubiquitous example of the simple pendulum modelled as a simple harmonic oscillator: the model incorporates a number of idealisations, such as the lack of air resistance (encountered by the bob), the lack of friction (between the string and that to which it is affixed), and of course the 'small oscillation assumption' which requires the angle of swing to be small (so that we $\operatorname{can} \operatorname{set} \sin \theta=\theta$ ). In this case there obviously is no isomorphism between the model and the target system (described appropriately).

However, with partial isomorphisms holding between partial structures we do not, of course, require complete structural identity between the model and the system. We recall that with a partial isomorphism, only certain of the $R_{i}$ of one partial structure stand in a one-to-one correspondence to certain of the $R_{I}^{\prime}$ of the other (da Costa and French 2003, p.49). Thus in the case of the simple pendulum, the idealised model can be said to represent the actual pendulum because only a partial isomorphism holds (in a sense to be clarified very shortly) between the model and the system (da Costa and French 2003, p. 102; see also Pincock 2005, p. 1254). This all sounds straightforward, with, of course, the

\footnotetext{
12 Weisberg himself also prefers a similarity based approach but defends one based on Tversky's contrast account (Weisberg 2013, pp. 143-155).

13 The other three desiderata are that on any such account a model must be maximally similar to itself and to any target that shares all of its properties; that the model-world relationship should accommodate 'rich' structures in terms of the kinds of properties involved and that the models should be tractable, in the sense that similarity judgments should be open to comparison and not dependent on any hidden or inaccessible features of the models concerned. All of which seem to be uncontentious requirements.
} 
caveat that the system itself - in this case the actual pendulum - is not a partial structure, so that the partial isomorphism holds not between the model and the actual system itself but between the former and the relevant data model or model of the phenomena (which would then incorporate the $R_{i}^{\prime}$ mentioned above) as captured via the hierarchy indicated previously (see French and Ladyman 1999).

However the following worry then arises: how are the idealisations captured by the partial structure ${ }^{14}$ ?

Let's begin with the small oscillation assumption. As every student of physics knows, when testing the formula for simple harmonic oscillation as applied to a simple pendulum, you have to restrict the angle of swing. Have it too high and the bob may even 'bounce' on the end of the string! So this will be a feature of the experimental set up and thus of the phenomenon to be accounted for. Thus, it will be captured by the $\mathrm{R}_{1}$ of the partial structure; that is, those relations that we know to hold in the system or phenomenon concerned. In that sense what we have in this case is not an idealisation per se, or at least not of the system being considered, although it might be regarded as such if we try to extend the application of our model outside of those situations where the assumptions hold (in which case the formula will fail!).

The air resistance and friction on the other hand will feature in the $R_{2}$, since they are being taken not to hold. In effect the model is being regarded in 'as if' terms: it is representing the pendulum as if there were no air resistance or friction. And as noted some years ago (da Costa and French 2003), the formalism of quasi- or partial truth, based on the partial structures framework, can then be used to underpin this claim that idealised models should be regarded as if they were true. Furthermore, as also noted (see also French and Ladyman 1998), it can be extended to cover not just this sort of case, but also that of idealisation terms, where these are thought of as idealising descriptions laid down within a theoretical context. To describe an electron as if it were a point particle is to describe it in terms of a bundle of properties which only have meaning within a model; thus the 'as if' character of such idealisation terms gets shifted to that of the embedding context. Our epistemic attitude to the latter can then be grounded in the notion of quasi-truth as well.

However, this is where Weisberg's worry comes in. Thus he notes that in many cases, '... it is the idealized features themselves that are supposed to be representations of the target's features and hence part of the explanation of the behaviour of such targets.' (op. cit. p. 141) But if, he continues, these features fall outwith that part of the partial structure that is connected via the given partial isomorphism to the other partial structure (Weisberg talks of 'non-isomorphic substructures in this regard) then in the terms of the partial structures programme, they cannot act as representations nor do any explanatory work.

Now, we have to be a little careful here. Consider again the simple pendulum: although the air resistance and friction are placed in the $\mathrm{R}_{2}$, as features that, for the purposes of the model, are taken not to hold of the system,

\footnotetext{
${ }^{14}$ Here we are talking about models that can be 'de-idealised'. It has been argued that there are certain idealisations - the 'thermodynamic limit' in statistical mechanics, for example - that cannot be dispensed with in this way and thus that play an essential explanatory role. However the nature of this role remains, at best, unclear with nothing to indicate how it can be situated in standard accounts of explanation (see Bueno and French 2012).
} 
so the latter is being modelled as if it were resistance-free and frictionless, the bob itself, for example, is still included in the model (in the $A$ ) and of course, within the scope, as it were, of the partial isomorphism. It is the $b o b$ that is the idealized 'feature' within the model (by being taken to suffer no air resistance) and this still both represents the actual bob and explains (within the limits of the idealisation) its behaviour. Thus the partial isomorphism still captures the representational relationship and thereby tracks the explanatory work being done.

This is a case of 'Aristotelian' idealization, where we 'remove' certain properties and consider what remains in isolation (Frigg and Hartmann 2012). What about Galilean idealization, where we keep the relevant properties but distort them? Weisberg gives the famous example of Schelling's model of racial segregation, which involves both forms of idealization; let us consider this in some detail:

Schelling constructed a simple yet elegant model to explain segregation whereby each agent prefers only that at least $30 \%$ of its neighbours be of the same kind (however defined) and said agent can move (even if only in a limited way - as in the squares on a chessboard). Schelling showed that even with such a weak preference, dramatic segregation will occur and this has been taken to imply that racial segregation need not be the result of racial prejudice. As Weisberg notes, the result is robust across various changes to the model, including, especially, changes to the preference and the spatial configuration (Weisberg op. cit., pp. 13-14) ${ }^{15}$. Here it is the idealized preferences (represented by utility functions) and spatial configuration that both represent the properties of actual people and do the explanatory work. Yet, he argues, if they are shunted away into the $R_{2}$, these representational and explanatory capacities cannot be captured by the partial structures approach.

The Schelling model is an example of what Gibbard and Varian call a 'caricature' model, where the assumptions of the model are chosen in order to isolate and exaggerate some feature of reality (Gibbard and Varian 1978). Paraphrasing their analysis ${ }^{16}$, some feature of social life is noted - in this case the tendency of neighbourhoods to become segregated. The scientist 'sets out' to construct an explanatory model but as the reality of racial segregation is complex, instead of trying to obtain the closest tractable approximation to the situation, she tries to tell a simple 'story' that captures the most salient features. The model then allows us to pose the question, what would happen if this story were true? (ibid., p. 674). In effect, Schelling constructed a model in which racial prejudice (apparently) played no role in segregation and in which the primary driving force is a mild preference. And he did this not because he thought it might be true or even approximately so but because he thought he could then isolate the effects he wanted to model (cf. ibid. fn 10).

\footnotetext{
15 Thus Pancs and Vrend (2007) show that even with a strict preference for perfect integration, segregation may result. Also, see Muldoon, Smith and Weisberg 2012.

${ }^{16}$ I am not suggesting that this was the heuristic route that Schelling actually took! However, the preface to (Schelling 2006/1978) does suggest that in much of his work (of which the Schelling model is only a small part) was inspired by striking examples or significant social phenomena of one kind or another.
} 
Thus, caricature models - as the name suggests ${ }^{17}$ - involve deliberate distortions of reality, and this is why they differ from idealizations like that of the simple harmonic oscillator. In the latter case, the aim is to distort as little as possible while achieving a degree of simplicity and tractability. Of course, excluding air resistance or friction is a distortion in a sense - after all, eventually, a real simple pendulum will stop swinging! - but we know from experimental practice that the latter can be considerably reduced and the former ignored for all relevant purposes. But in the case of caricature models, the distortion is central with the aim of isolating a certain factor, or ruling out others ${ }^{18}$.

What then is the value of such models? One answer has to do with their robustness, in the sense that their conclusions do not depend on the details of the core assumptions. Thus in the case of the Schelling model, as Weisberg has noted, we can change the preferences, even quite dramatically, or the spatial configuration and the result still obtains. The model appears to be robust indeed, claims that the model has a physical analogue have been dismissed as not advancing our understanding of the social dynamics of segregation on the grounds that the mathematical basis of the model and simulations based on it have already done the job (Clark and Fossett 2008) - and has become one of the most widely cited and highly regarded models in the social sciences in general ${ }^{19}$.

Now, what about Weisberg's worry? Let me reiterate that the Schelling model is not a straightforward idealisation, akin to the simple pendulum, but a 'caricature' model in which an 'impression' is given of racial segregation, not by modelling it and its underlying causes directly, but by showing how it may be the unintended 'macro-behavioural' consequence of 'micro-motivations', in this case - fairly mild preferences. Thus although the explanandum might well be characterised as a social phenomenon as given in this context, it is entirely impressionistic in that it is not presented as racial segregation in a certain city, or district or even across the US but simply as unspecified segregation, abstractly understood (indeed, as Schelling himself emphasises (in 2006/1978 and elsewhere) he was interested in segregation as a quite general phenomenon, whether in terms of race, gender or even between officers and enlisted men (and now, presumably, women) in the armed forces).

Furthermore, the explanans - the model itself - is quite simple in terms of what is assumed. Weisberg suggests these assumptions must be regarded as part of the 'non-isomorphic substructure' and hence, on the partial structures view, cannot be seen as doing any explanatory work. In contrast, I would argue that we should not assign them to the $\mathrm{R}_{2}$, as that would indicate that we know that they

\footnotetext{
17 Interestingly, Gibbard and Varian explicitly compare models to pictorial representations: econometric models - of the kind that run of computers and are used to make economic forecasts are likened to photographs (perhaps, given recent events, really bad, out of focus photographs ... of the economist's thumb); what they call 'approximations', which we might term idealised models, are akin to pencil drawings and caricature models are like, well, caricatures (1978, p. 665). And as they note, a given model may evolve from a caricature to an approximation or even to an econometric model.

18 Thus - following on from the previous footnote - the distortion illuminates a certain feature of reality, just as a pictorial caricature does (ibid., p. 676).

${ }^{19}$ Frigg and Hartmann (2012) suggest that it is controversial whether such caricature models can be regarded as informative representations of their target systems, citing (Reiss 2006) However, Reiss only briefly mentions these models in the context of arguing that they cannot be used to establish the existence of Cartwrightian capacities in the domain of social science.
} 
definitely do not hold (as the colours of a billiard ball do not hold for gas atoms in the classic example - see da Costa and French 2003). But, first of all, given that we are talking about a caricature of a quite abstract phenomenon rather than a specific system, we know no such thing. Secondly, and relatedly, we simply do not know whether these assumptions hold for real 'systems', that is, actual neighbourhoods. Again, that's not the point.

And that its not the point means that these assumptions should not simply be represented as part of the $\mathrm{R}_{3}$ - those relations for which we do not know whether they hold or not. Although that is true of actual neighbourhoods or groups of people, it is not the case for the distorted feature of reality in question. In this case, we know that the assumptions yield the segregation and thus they hold in this context. Thus they should be placed in the $\mathrm{R}_{1}-$ i.e. very much not part of the 'non-isomorphic substructure' - but the model, of course, should be viewed as offering an 'as if' story (see above) in the sense of answering the question, what would happen if it were true that people preferred $1 / 3$ of their neighbours to be the same race? Answer: segregation! As already suggested, the theory of quasi-truth can then be used to formally underpin such 'as if' claims ${ }^{20}$. And as Weisberg himself notes (op. cit. pp. 118-119), although such models might be used in a 'target-directed' manner - in the sense of being used to explain the segregation in an actual neighbourhood or city - Schelling himself was interested in how it might be possible for segregation to result without collective preferences for it. What we have here is a 'how-possible' kind of explanation (ibid.).

But of course that is not the whole story. The worry has to do with whether the partial structures approach can adequately capture the 'work' that the model is doing. Now if that work were only encapsulated in the above, Schelling's model would be interesting but not, perhaps, as impressive as it is. However, as noted previously, the model is robust under a range of alternative assumptions and this is where much of its value lies - one can tweak the core assumptions in various ways and weaken them quite considerably, and segregation still results. From the perspective of the model as offering an 'as if' story, one can take these alternatives as being 'stored', as it were, in the $\mathrm{R}_{3}$ - after all, from this perspective, we do not know if they hold, not of the actual social 'systems' but of segregation as distorted and broadly understood in this manner. Thus we can understand robustness analyses as 'promoting' various of these alternatives from the $\mathrm{R}_{3}$ to the $\mathrm{R}_{1}$ - effectively giving us different variants of the model, again, regarded as 'as if' (cf. Weisberg op. cit., p. 158) - and then determining whether segregation still results. That it does, under a wide array of alternatives, has been taken by many social scientists to support, in some sense,

\footnotetext{
20 It also helps respond to the worry about how strictly false models, containing such idealizations, can still explain - the answer is that although false, insofar as they work, whether in an explanatory sense or otherwise, they can be regarded as partially or quasi-true (da Costa and French 1990). Whether this corresponds to an appropriate form of approximate truth is another matter. Consider the model of a solenoid in which the magnetic field is taken to extend to infinity - this is not approximate to any real life situation (thanks to James Fraser for this example). However insofar as this is so only by virtue of the fact that infinity is not approximate to any finite quantity, one might respond either by treating such as special cases, depending on one's view of infinity (i.e. a constructivist would have very definite views on such examples!) or by noting that just as physicists talk, perhaps loosely, of bringing in a test charge 'from infinity' so we can talk of dialling down the field 'from infinity'.
} 
the model; or perhaps better, to support the hypothesis that segregation may result via the expression of quite mild preferences and is not necessarily due to (overt) prejudice.

How should we cash out this notion of support? One option is via some notion of confirmation. Many philosophers of science, however, have been resistant to such suggestions ${ }^{21}$. The grounds for this scepticism have to do with the lack of further evidence involved, since it is essentially the same result or general phenomenon that is being appealed to in each case, or iteration, supporting the robustness claim. Now one might immediately object that in the case of the Schelling model, there is of course no 'further evidence' since what we have is a caricature of social reality to begin with! Nevertheless, we should resist the shift from that exclamation to the dismissal of the practice of social scientists themselves in setting such considerable store by these robustness results.

Weisberg himself suggests that robustness offers '... low-level confirmation, confirmation of the fact that certain mathematical structures can adequately represent properties of target phenomena.' (2006, p. 740). In the Schelling case, the robust property or phenomenon we are concerned with is, obviously, segregation. By varying the assumptions made we obtain an array of models, all of which yield this phenomenon (hence, its robustness). We then extract the common structure from that array which we take to be responsible for this phenomenon (Weisberg 2013, p. 158) and form a 'robust theorem': ceteris paribus, if individuals' decisions about where to live are guided by the kinds of considerations encapsulated in the models' assumptions, then segregation will result (see Weisberg ibid., p. 168, for a more precise expression). According to Weisberg, robustness analysis does not confirm such robust theorems (so in that sense he agrees with the sceptics), but what it does is identify hypotheses ' ... whose confirmation derives from the low-level confirmation of the mathematical framework in which they are embedded.' (ibid. p. 170).

So, what we have is a general phenomenon, segregation, expressed quite abstractly and non-specifically, that can be accommodated by each of an array of models, each incorporating an alternative assumption about, for example, individuals' preferences but all manifesting a common structure. From the settheoretic perspective, a sub-structure, expressing, again quite generically, this phenomenon, can be regarded as embedded in each of these models and to that extent can be taken as confirming them, if only weakly. That the phenomenon is adequate, if not empirically then in some sense, to all the models could be seen as a kind of underdetermination. From this perspective, what robustness analysis rests on is a kind of 'ontological retreat' to the common structure, which is where we should place our epistemic emphasis in each case. More importantly, there appears to be nothing in Weisberg's account that falls outside the partial structures approach: the models can be regarded as of the 'as if' kind, corresponding to 'how-possibly explanations', just as other forms of idealisation

\footnotetext{
${ }^{21}$ See Woodward (2006), who also gives a useful classification of different forms of robustness. What we have in the Schelling case would appear to fall under what he calls 'derivational robustness', whereby '...an assumption is adopted about the value of the parameter and this is used, in conjunction with other theoretical assumptions, to derive some range of observed phenomena. Investigations are then made whether, given other values of the parameter, but the same theoretical assumptions, the same conclusions can be derived.' (ibid., p. 233)
} 
are according to this approach (French and Ladyman 1998; da Costa and French 2003) and the explanatory work that they do can be captured by initially placing the relevant assumptions in the $\mathrm{R}_{3}$ and then 'promoting' each one to the $\mathrm{R}_{1}$ to yield a different 'as if' model in each case. What this then allows us to do via the robustness analysis, as already indicated, is to identify the common structure, which can be represented set theoretically as sitting in the $\mathrm{R}_{1}$. And what is thus confirmed is the 'representational capacity' of this structure (Weisberg 2013, p. $169)^{22}$.

There is a further concern, however, that has been expressed by Pincock, to the effect that the partial structures approach must treat idealisations as nothing more than a series of approximations, but because the latter are too crude to discriminate among different idealizations, the approach cannot rank or evaluate them in a way that meshes with scientific practice (Pincock 2005). Consider again the case of the simple pendulum, treated as a simple harmonic oscillator (that is, with air resistance and frictional effects ignored): as is well known, the motion of the pendulum can be represented in the appropriate phase space as an ellipse, determined by the length of the pendulum, the gravitational constant and the initial state of the pendulum (ibid., pp. 1250-1251). If air resistance or friction is included then the phase space trajectory spirals into the origin, of course. Our (total or complete) model will thus consist of the domain of possible states - as represented in this phase space - and a family of relations representing all the 'simple harmonic' trajectories generated by all the possible initial states of the pendulum. Given the idealizations (including the small oscillation assumption) these trajectories will not be isomorphic to actual trajectories but they can be taken to be partially isomorphic (ibid., p. 1254).

This suggests that in the mathematical description of the trajectories a further 'error' term needs to be introduced to reflect the 'looseness' of the idealization, yielding a series of tubes in phase space (ibid., p. 1256). As Pincock emphasises, the assumptions behind this move (ignoring air resistance, keeping the oscillations small etc.) are all well motivated. However, we can easily generate the same tubes from a different mathematical description via moves that are not so well motivated or indeed, not motivated at all (ibid.). From the perspective of the partial structures approach, which looks only at the end result as it were and then insists that a partial isomorphism can be taken to hold between these tubes and the actual trajectory, there appears to be no way to discriminate between the well-motivated idealisations and the poorly motivated ones. In particular, appealing to further model-model relations in the hierarchy outlined above will not help, because the same mappings will hold for both the well motivated model and the poorly motivated alternative (ibid., p. 1258).

As a result,

“... we cannot ignore how or why a mathematical model is introduced if we are to give a reasonable account of representation and accuracy in terms of partial isomorphisms. In particular, our judgments about the appropriateness of an

22 Paternotte and Grose (forthcoming) argue that not all cases of robustness can be accommodated by Weisberg's analysis. They use examples from evolutionary game theory to show that in such cases there is no common structure, yet robustness may be justified in cases of phenomena that follow from multiple initial conditions or are multiply instantiated. 
idealized model depend not just on the trajectories that we end up with, but how we cooked up these particular trajectories in the first place." (ibid., p. 1257)

Now, I would certainly, and obviously, agree that our judgments as to which idealisations to incorporate in a model depend on a variety of factors that will be external to the model itself. Indeed, how could they be otherwise? Consider, again, the small oscillation assumption, which is dependent on the mathematical result that for small $\theta \sin \theta \sim \theta$, allowing us to more easily solve the relevant differential equation and thus contributing to the model's mathematical tractability. When it comes to friction and air resistance, we know from empirical studies that we can ignore these for most situations - of course, if we were to swing the pendulum in water or add some restrictive material at the point where the string is affixed, then we would not get the expected relationship between the period and length and our model would fail. So, again, I agree that we cannot ignore how or why the model was introduced but the factors laying behind that introduction will typically be those that fall outside of the model itself, not least because they may include empirical features of the situation. In other words, we should not have expected the force of these factors to have been captured by the partial structures formalism alone, or indeed any other such formal framework.

The point has been made before with regard to heuristic factors involved in theory or model construction (see da Costa and French 2003 Ch. 4) ${ }^{23}$ and can be extended here: we should not expect formal representations of theories or models to incorporate heuristic moves, the judgments of scientists and such like. To insist that we must and then claim that attempts to do so fail is to attempt a knock down argument against a self-evidently absurd position. Thus, our judgments about the appropriateness of an idealized model such as the simple pendulum should not, of course, depend only on the phase space trajectories we end up but should involve consideration of the factors, empirical and otherwise, that led to the relevant assumptions being made. Of course, insofar as these assumptions are included in the model, the factors concerned are also manifested in that model, in a sense, but in order to rank it as better or worse than an alternative, we obviously need to consider the factors themselves (e.g. is the lack of friction empirically well grounded). Thus just as we should not expect the formalism of the partial structures approach to capture the confirmation of models or theories, so we should hold no similar expectation with regard to the ranking of idealizations (or at least, not with regard to that aspect of the ranking that depends on factors external to the formalism).

With the above to hand, we can dispense with the other objections more straightforwardly. Thus Weisberg raises a similar concern to Pincock's in arguing that partial isomorphism based accounts fail to account for 'context': although he acknowledges that since partial isomorphisms come in degrees, it could be argued that '... contextual factors dictate the determination of when a particular partial isomorphism is good enough for the purpose at hand' (2013, p. 141), he maintains both that this determination would not be part of the partial structures account of the model-world relation and that no separate account of how to make such judgments has been given.

Now it could be argued that context can be brought into play to determine

${ }^{23}$ Indeed, Pincock acknowledges this point (op. cit., p. 1253). 
when a particular partial isomorphism is appropriate, as indeed it has (da Costa and French 2003)! And it is correct, of course, that this determination is not 'internal' to the partial structures formalism - again, how could it be? One might argue that an account that can incorporate such factors would be preferable but in this specific case, if 'context' is internalised, as it were, one might wonder in what sense it can still be regarded as contextual. Of course, the formalism should be flexible enough to adapt to different contexts, and I believe that the partial structures approach is, but I also do not think that it should be tied to a specific account of the impact of contextual factors, just as it should not be tied to specific accounts of confirmation, Bayesian or otherwise. Some indications of how accounts of these various judgments might mesh with the approach have in fact been presented (ibid.) but given that preference for one such account over another will involve reasons that go beyond the approach itself, I would argue that a degree of distance or, better, flexibility should be maintained.

Relatedly, Weisberg also argues that although the partial structures approach could allow for the construction of some kind of metric that would meet the 'scalar' desideratum, this has not in fact been attempted. However, such a possibility was explicitly mentioned in one of the very first presentations of this approach (da Costa and French 1990), where it was noted that the degree to which a model might be said to 'approximate' a theory could be measured by the difference between the relevant $R_{k}$ and $R_{k}{ }_{k}$ on some scale, along the lines previously indicated in (Redhead 1980). This can easily be extended to accommodate the kinds of concerns that motivated this desideratum. And of course, the terms in which such differences are measured will depend - once again - on factors outwith the partial structures approach, which is as it should be. Certainly, insofar as these differences are representable on some scale or other, this approach meets the requirement. As to why its advocates have not themselves developed such a metric, its simply because they have not felt any need to, given the issues they have been concerned with. But if anyone else wants to take this on, then have at it!

Finally, and perhaps most problematically in some senses, '[by] definition, no model-theoretic accounts can meet the QUALITATIVE desideratum' (Weisberg 2013, p. 141). However, it is not entirely clear why, '[b]y definition' such accounts cannot meet this requirement. One thought might be that because they are grounded in set theory, these accounts can only make quantitative comparisons between models and the world. If so, then given that many models, particularly outside of the physical sciences, involve only qualitative

comparisons - the Schelling model being precisely one such (ibid., p. 136) - then model theoretic accounts would be deficient for not being able to accommodate such models. But insofar as the $\mathrm{R}_{\mathrm{i}}$ can capture qualitative as well as quantitative relations, this is no problem at all (thus, the partial structures approach can be extended beyond the physical sciences to, for example, biology and economics; again see da Costa and French 2003 or French 2014 Ch. 12). Returning to the Schelling model, as Weisberg notes (op. cit., p. 136), here 'one will compare the fact that the model has racially segregated clusters to the fact that the city has racially segregated clusters'. The relevant racial segregation is then straightforwardly represented in terms of the $\mathrm{R}_{\mathrm{i}}$ and the issue comes down to comparing it with what is observed in reality. One can imagine borderline cases where it is not immediately clear whether there is the kind of segregation in the 
city that is presented by the model, and one might then impose some kind of metric on one's observations and move to a model of the phenomena, but even in straightforward cases there is no requirement that the relations manifested by segregation in the city have to be quantitatively represented to be comparable with those in the model.

This brings us to the issue of the model-world relationship and the second, alternative reason why model-theoretic accounts cannot meet this desideratum. Thus Weisberg insists that it is because '[s]uch accounts can only compare structure to structure' (ibid., p. 141). Now, let me say first of all that there is a sense in which this is correct: strictly speaking partial isomorphisms can only be defined between and thus can only be taken to hold between set theoretic structures. But this is an old concern that has since been addressed (French and Ladyman 1999) - how any of our representations, linguistic, modeltheoretic, artistic or otherwise, relate to the world is of course a fundamental philosophical problem but in this context, we can either acknowledge that in most scientific cases what we're talking about is the relationship between theoretical models, empirical substructures and models of the phenomena etc., which of course can all be represented set-theoretically (but which just pushes the issue back to that of the relationship between the model of the phenomena and the phenomena itself) or accept that talk of a partial isomorphism holding between a model and the world is a façon de parler that allows us to capture the assertion of a primitive or intuitive similarity between the Schelling model, say, and racial segregation in some city of other. Of course, even in this case, its not as if we have that racial segregation to hand, as it were, or can observe it in such a way that we can relate it to the model, even qualitiatively - what we would have is the graphical representation of the outcome of the Schelling model and a similarly graphical representation of the racial segregation in some city and whether we say they are similar by just eyeballing the two or via some more precise technique, there is nothing here that can't be capture set-theoretically.

Finally, however, Weisberg seems to place further weight on this claim that the model-theoretic approach only relates structures to structures by suggesting that qualitative properties cannot be structural and this is why the desideratum cannot be met (ibid. p. 141). Here the idea is that qualitative properties may be monadic and not relational and hence can't be captured via the model theoretic approach. But that's simply not the case - the $\mathrm{R}_{\mathrm{i}}$ are perfectly general and may include monadic features possessed by each element in the relevant $A^{24}$.

Thus I would argue that all of Weisberg's (and Pincock's) concerns can be met, either by paying due attention to the relevant features of the partial structures framework or by acknowledging the role of factors external to that framework. This aspect of scientific practice, then, presents no obstacles to the semantic approach.

\footnotetext{
24 Weisberg suggests that many proponents of the partial structure approach are structural realists who would deny that qualitative features are relevant to scientific enquiry (ibid., p. 141). But of course one could be a structural realist with regard to the most fundamental properties, in physics say, and still accept that features of systems at 'higher' levels or in different domains might best be described in non-relational terms.
} 


\section{Conclusion}

I began this paper by noting how recent criticisms of the semantic approach have been framed by a concern regarding the identity conditions of scientific theories that has fed into the underlying assumption that according to this approach theories should be identified with families of models. I have tried to suggest that this assumption should be abandoned, that we should give up on trying to specify such identity conditions and that we should regard the semantic approach instrumentally as a useful set of devices that philosophers of science can deploy to further our aims. Such aims will include, of course, that of appropriately representing those features of scientific practice that we are interested in. Some of those features might be labelled 'theories' or 'models' or 'research programmes' but, again, we should be wary of taking them to be delineated too tightly. In particular, they will typically be open-ended and interrelated in often complex ways. Given this, I have pressed, the partial structures variant of the semantic approach offers the best meta-level representation of these features.

A possible obstacle to this insistence is the role of idealisations in science, which, Weisberg and Pincock have strongly argued, cannot be accommodated in this framework. I have tried to respond that they can but this will involve paying attention to certain factors laying beyond that framework. In a sense, "twas ever thus' but it is important to acknowledge the limitations of formal approaches they cannot capture, for example, the nature and role of heuristic motivations, although they can certainly be made compatible with them (again, see da Costa and French 2003 Ch. 4).

Such motivations lie, of course, within scientific practice itself and although one can conceive of ways in which they might be (non-formally) captured at the meta-representational level, that acknowledgment further suggests that accommodating that practice within one's philosophy of science is going to involve a complicated to-and-fro dance between the particular instrumental device that one has pragmatically chosen and those factors within the practice that will lie beyond its formal grasp. And of course, in so moving back and forth, the formal structures one constructs may in turn inform the features of practice one started out by representing. Consider Suppes' work in the foundations of psychology for example, particularly with regard to learning theory and cognitive psychology. There his axiomatizations in terms of certain primitive concepts drawn from psychological practice can be seen as helping to shape or even crystallize the relevant theories (Batchelder and Wexler 1979). Such examples may be few and far between (others may be drawn from the collaboration between physicists and philosophers on Bohm theory or at the 'coalface' of the development of forms of quantum gravity) but they suggest interesting ways in which philosophy may interact with or even impact upon science.

Less contentiously perhaps, this general stance towards object-level scientific practice on the one hand and meta-level philosophy of science, might offer new avenues for thinking about the relationship between science, and its history, and philosophy of science. At the very least what we seem to have is less a case of the former acting as evidence, whether confirmatory or falsificatory, for the latter, and more that of an iterative process, hopping between levels, in 
which what we take to be the relevant features to be accommodated is itself shaped by the approaches and consequent devices we adopt as philosophers. Dropping the assumption that there are definite things called theories and adopting an instrumental stance towards the semantic approach (and others) may thus lead to a more integrated approach to science, the history of science and the philosophy of science more generally.

\section{References}

Batchelder, W.H. and Wexler, K. (1979). Suppes' Work in the Foundations of Psychology. In E.J. Bogdan (ed.), Profiles: Patrick Suppes (pp. 149-186). Dodrecht: D. Reidel.

Bueno, O. (1997). Empirical Adequacy: A Partial Structures Approach. Studies in History and Philosophy of Science 28, 585-610.

Bueno, O. (2000). Empiricism, Mathematical Change and Scientific Change. Studies in History and Philosophy of Science 31, 269-296.

Bueno, 0. (2016). Belief Systems and Partial Spaces. Foundations of Science 21, 225-236.

Bueno, O., French, S., and Ladyman, J. (2002). On Representing the Relationship between the Mathematical and the Empirical. Philosophy of Science, 69, 497518.

Bueno, O. and French, S. (2012). Can Mathematics Explain Physical Phenomena? The British Journal for the Philosophy of Science 63, 85-113

Bueno, O. and French, S. (forthcoming), Applying Mathematics: Immersion, Inference, and Interpretation

Clark, W.A.V. and Fossett, M. (2008), Understanding the social context of the Schelling segregation model. Proceedings of the National Academy of Sciences 105, 4109-4114.

Curiel, E. (2014). Classical Mechanics Is Lagrangian; It Is Not Hamiltonian. British Journal for the Philosophy of Science 65, 269-321.

da Costa, N.C.A. and French, S. (1990). The Model-Theoretic Approach in the Philosophy of Science. Philosophy of Science 57, 248 - 265.

da Costa, N.C.A. and French, S. (2000). Theories, Models and Structures: Thirty Years On. Philosophy of Science 67 (Proceedings), S116-S127. 
da Costa, N.C.A. and French, S. (2003), Science and Partial Truth: A Unitary Approach to Models and Reasoning in Science. Oxford: Oxford University Press

Fraser, D. (2011). How to Take Particle Physics Seriously: A Further Defence of Axiomatic Quantum Field Theory. Studies in History and Philosophy of Modern Physics 42, 126-35.

French, S. (2014). The Structure of the World. Oxford: Oxford University Press.

French, S. (forthcoming). (Structural) Realism and its Representational Vehicles. Forthcoming in Synthese (DOI 10.1007/s11229-015-0879-x)

French, S. and Ladyman, J. (1998). A Semantic Perspective on Idealisation in Quantum Mechanics. In N. Shanks(ed.), Idealization IX: Idealization in Contemporary Physics: Pozna'n Studies in the Philosophy of the Sciences and the Humanities, (pp. 51-73). Rodopi.

French, S. and Ladyman, J. (1999). Reinflating the Semantic Approach. International Studies in the Philosophy of Science 13, 99-117.

French, S. and Saatsi, J. (2006). Realism about Structure: The Semantic View and Non-linguistic Representations. Philosophy of Science (Proceedings) 78, 548-559, 2006.

Frigg, R. (2006). Scientific Representation and the Semantic View of Theories. Theoria 55, 37-53.

Frigg, R. and Hartmann, S. (2012). Models in Science. The Stanford Encyclopedia of Philosophy (Fall 2012 Edition), Edward N. Zalta (ed.), URL = <http://plato.stanford.edu/archives/fall2012/entries/models-science/>.

Gibbard, A. and Varian, H.R. (1978). Economic Models. The Journal of Philosophy $75,664-677$

Glymour, C. (2013), Theoretical Equivalence and the Semantic View of Theories. Philosophy of Science 80, pp. 286-297

Gooday, G. and Mitchell, D. (2013). Rethinking 'Classical Physics. In Jed Z. Buchwald and Robert Fox (eds.), The Oxford Handbook of the History of Physics. Oxford: Oxford University Press.

Halvorson, H. (2012). What Scientific Theories Could Not Be. Philosophy of Science 79, 183-206

LeBihan, S. (2012). Defending the Semantic View: what it takes. European Journal for the Philosophy of Science 2, 249-274.

Muldoon, R., Smith, T. and Weisberg, M. (2012). Segregation That No One Seeks. Philosophy of Science 79, 38-62. 
North, J. (2009). The "Structure" of Physics: A Case Study. Journal of Philosophy 106, 57-88.

Pancs, R. Vriend, N.J. (2007). Schelling's spatial proximity model of segregation revisited. Journal of Public Economics 91, 1-24

Paternotte, C. and Grose, J (forthcoming). Robustness in evolutionary explanations: a positive account. Forthcoming in Biology and Philosophy.

Redhead, M.L.G. (1980). Models in Physics. British Journal for the Philosophy of Science 3, 145-163.

Reiss, J. (2006). Beyond Capacities. In Luc Bovens and Stephan Hartmann (eds.), Nancy Cartwright's Philosophy of Science. London: Routledge.

Pincock, C. (2005). Overextending Partial Structures: Idealization and Abstraction. Philosophy of Science (Proceedings) 72, 1248-1259.

Schelling, T.C. (2006/1978) Micromotives and Macrobehaviour, W.W. Norton

Suppe, F. (1989). The Semantic View of Theories and Scientific Realism. Urbana and Chicago: University of Illinois Press.

Thomson-Jones, M. (2006). Models and the Semantic View. Philosophy of Science $73,524-35$.

van Fraassen, B. (1989). Laws and Symmetry. Oxford: Oxford University Press.

van Fraassen, B. (2014). One or Two Gentle Remarks About Hans Halvorson's Critique of the Semantic View. Philosophy of Science 81, 276-283.

Vickers, P. (2013). Understanding inconsistent science. Oxford: OUP.

Wallace, D. (2011). Taking Particle Physics Seriously: a Critique of the Algebraic Approach to Quantum Field Theory. Studies in the History and Philosophy of Modern Physics 42, 116-25.

Weisberg, M. (2006). Robustness Analysis. Philosophy of Science 73, 730-742.

Weisberg, M. (2013). Simulation and Similarity: Using Models to Understand the World. Oxford: Oxford University Press

Wilson, M. (2014). What is Classical Mechanics Anyway?

http://www.philosophy.pitt.edu/sites/default/files/whatisclassicalmechanicsan yway.pdf (an abridged version can be found in The Oxford Handbook in Philosophy of Physics, R. Batterman, ed, Oxford: Oxford University of Press). 
Woodward, J. (2006). Some varieties of robustness. Journal of Economic Methodology 13, 219-240. 